\title{
Low-dose UVB irradiation prevents MMP2-induced skin hyperplasia by inhibiting inflammation and ROS
}

 \\ ${ }^{1}$ Department of Dermatology, The Second Affiliated Hospital of Harbin Medical University, Harbin, Heilongjiang 150086; \\ ${ }^{2}$ Department of Pathology, Heilongjiang Provincial Hospital, Harbin, Heilongjiang 150036; ${ }^{3}$ Department of Dermatology, \\ The First Affiliated Hospital of Harbin Medical University, Harbin, Heilongjiang 150001, P.R. China
}

Received April 23, 2015; Accepted June 11, 2015

DOI: $10.3892 /$ or.2015.4072

\begin{abstract}
Skin cancer is one of the most common types of malignancy in the world. UV radiation is known as the primary environmental carcinogen responsible for skin cancer development. However, UV radiation is a ubiquitous substance existing in the environment and the physiological effect of UV radiation is consistently ignored. Therefore, in the present study, the physiological effect of UV radiation on inhibition of skin cancer was investigated. Normal mouse skin was processing by no pre-radiation or pre-radiation of low-dose UV before a medium or high dose of UV radiation. We found that the low-dose pre-radiated mouse skin tissue exhibited low skin inflammation, skin ROS production and consequently low skin epithelial hyperplasia after the medium-dose UV radiation compared with the no pre-radiated mouse. However, this inhibition was not indicated in the high-dose UV radiation group after low-dose pre-radiation. Furthermore, western blot analysis and gelatin zymography showed low expression and activation of MMP2 in the skin tissues processed following medium-dose radiation, but not in tissues treated with high-dose radiation after a low-dose pre-radiation. Further investigation of MMP2 inhibitors of TIMP2/TIMP4 showed an upregulated TIMP2 expression, but not TIMP4. Collectively, these data indicate that low-dose pre-radiation attenuates the skin inflammation and ROS production induced by medium-dose UV radiation and also elevates TIMP2 to withstand MMP2, therefore suppressing skin hyperplasia. The present study indicates a novel concept or prophylactic function of moderate UV radiation as a preventative strategy.
\end{abstract}

Correspondence to: Professor Jikui Xiong, Department of Dermatology, The Second Affiliated Hospital of Harbin Medical University, 246 Xuefu Road, Nangang, Harbin, Heilongjiang 150086, P.R. China

E-mail: xiongjikui@126.com

Key words: skin cancer, low dose UV radiation, skin inflammation, skin ROS production, skin epithelial hyperplasia, MMP2, TIMP2/ TIMP

\section{Introduction}

Skin cancer has become a prevalent social and public health issue due to the fact that the incidence and mortality rates of skin cancer, including melanoma and non-melanoma skin cancers (NMSCs), consisting of basal cell cardinoma (BCC) and squamous cell carcinoma (SCC) are rising in many countries $(1,2)$. Skin cancer patients are associated with higher UV radiation exposure (3) and UVB susceptibility was reported to be significantly higher in skin cancer patients (4). In general, UV irradiation induces DNA damage, repair, skin inflammation and sunburn, gene mutation, post-inflammatory immunosuppression and eventually oncogenic pathway activity and is considered to be a major aetiological factor for skin carcinogenesis $(5,6)$.

UVB exposure induces cutaneous inflammation. A single exposure to UVB may result in an acute inflammatory response, which is characterized by erythema (redness) and edema (swelling) due to increased vascular flow and vascular permeability $(7,8)$. An inflammatory microenvironment aids in the proliferation and survival of malignant cells, promotes angiogenesis and metastasis, subverts adaptive immune responses and alters responses to hormones and chemotherapeutic agents, all of which promote the development of tumors $(9,10)$. Inflammation is always accompanied by oxidative stress induced by reactive oxygen species (ROS) accumulation. ROS are believed to activate proliferative and cell survival signaling that alter apoptotic pathways that may be involved in the pathogenesis of a number of skin disorders. ROS act largely by driving several important molecular pathways that play important roles in diverse pathologic processes including inflammatory responses (11). Inflammation and ROS are inseparable and interact in the skin cancer process.

However, UV irradiation in sunlight is the most ubiquitous physical substance in our natural environment. Low-dose UVB induces non-inflammation and normal non-inflammatory apoptosis (12), which is a physiological process and conserved feature for the non-inflammatory removal of cells in order to protect organisms (13). Low-dose UV irradiation is also well known to be the primary source of Vitamin D3 synthesis, which acts as a sunscreen to prevent skin and various other types of cancer (14-16). Skin cancer, in the United States, is reported to account for 20 to $30 \%$ of all neoplasms in Caucasians, 2 to 
$4 \%$ of all neoplasms in Asians and 1 to $2 \%$ of all neoplasms in Blacks and Asian Indians (17-19). This fact indicates that skin cancer is less common in persons with skin color other than light-skin. Thus, melanin pigment is a UV screen that protects some deleterious sunlight compositions $(20,21)$. Although the skin coloration in dark-skinned ethnic groups is the result of long-term evolution based on resistance and prevention of UVB irradiation-induced skin injury $(22,23)$, in the short term, skin coloration namely pigmentation is due to solar irradiation. These studies indicate that a low dose of UV irradiation plays a protective role in skin cancer.

However, skin cancer still presents greater morbidity and mortality in colored-skinned individuals (17). It appears that the morbidity and mortality of skin cancer is not paralleled with incidence, since UVB irradiation depletes heavily pigmented skin of Langerhans cells (20). Once limited pigmented skin is depleted, the skin cancerization rate greatly increases. Therefore, the skin cancer protective role of melanin pigment is limited or UV-relative, but not an absolute prevention for skin cancer.

Collectively although excess UV irradiation certainly induces skin cancer, low-dose UV irradiation exhibits a protective role to prevent skin cancer yet is limited: i) to the most initial stage of cutaneous lesions or ii) to relative slight cutaneous lesions. However, we do not know how low-dose UV irradiation prevents these cutaneous lesions.

In the present study, we performed low-dose UVB pre-irradiation and then subsequent medium- or high-dose UVB irradiation. We found that low-dose UVB pre-irradiation inhibited medium-dose-induced skin inflammation, ROS production and initial keratinocyte hypertrophy while high-dose UVB irradiation did not induce change. This inhibition was associated with the balance of MMP2 and its inhibitor.

\section{Materials and methods}

Mice and $U V$ irradiation. In the present experiment C57BL/6N mice were used and all the mice were purchased from Biocytogen Co., Ltd. (Beijing, China). The dorsal hair was removed by a depilatory in an area of $4 \mathrm{~cm}^{2}$, and all mice were divided into $3 \mathrm{UV}$ irradiation groups and 1 control group ( $n=8 /$ group). The UV irradiation groups were exposed to low-, medium- and high-dose $\left(100,350\right.$ and $\left.800 \mathrm{~mJ} / \mathrm{cm}^{2}\right) \mathrm{UV}$ irradiation (FL20S. E; Toshiba, Tokyo, Japan) for $5 \mathrm{~min} /$ day, 5 days every week for 4 weeks from the age of 8 weeks. Low-dose pre-irradiation was performed from the age of 6 weeks and then formal irradiation was carried out. All mice were fed normal rodent chow and had ad libitum access to water. The present study was approved by the Ethics Committee of the Harbin Medical University, Harbin, China.

Cell culture and detection of intracellular ROS. Intracellular accumulation of ROS was estimated using the fluorescent dye $\mathrm{H}_{2}$-DCFDA (Sigma-Aldrich, Shanghai, China), which is converted to a membrane-impermeable and highly fluorescent compound, dichlorofluorescein diacetate (DCF), in cells in the presence of ROS (24). The epidermal keratinocyte cell line HacaT (Biomics Biotech, Jiangsu, China) was used in the in vitro experiment as a source of keratinocytes. Cells
Table I. The primers used for the RT-PCR analysis.

\begin{tabular}{lll}
\hline IL-1 $\alpha$ & F: & 5'-GAAGAGACGGCTGAGTTTCAGTG-3' \\
& R: & 5'-CTGGTAGGTGTAAGGTGCTGA-3' \\
IL-1 $\beta$ & F: & 5'-TCATTGTGGCTGTGGAGAAG-3' \\
& R: & 5'-CAGTTGTCTAATGGGAACGT-3' \\
IL-6 & F: & 5'-ACAACCACGGCCTTCCCTACTT-3' \\
& R: & 5'-CACGATTTCCCAGAGAACATGTG-3' \\
SOD1 & F: & 5'-ACTAGTATGGCGATGAAAGCGGTG-3' \\
& R: & 5'-GGATCCTGTTTACTGGGCAATCCC-3' \\
Catalase & F: & 5'-ATGTCGGACAGTCGGGAC-3' \\
& R: & 5'-GACTGTGGAGAACCGAAC-3' \\
18s & F: & 5'-GTAACCCGTTGAACCCCATT-3' \\
& R: & 5'-CCATCCAATCGGTAGTAGCG-3'
\end{tabular}

Total RNA was extracted from exposed dorsal skin tissues and relative mRNA was normalized to the 18 s rRNA. F, forward; R, reverse.

were cultured in complete DMEM (L-glutamine, pyridoxine hydrochloride, sodium pyruvate and FCS) at $37^{\circ} \mathrm{C}$ in a $5 \%$ $\mathrm{CO}_{2} / 95 \%$ air humidified atmosphere. UV irradiation groups were exposed to low-, medium- and high-dose (10, 35 and $80 \mathrm{~mJ} / \mathrm{cm}^{2}$ ) UV irradiation as reported previously (12). After three days of low-dose UV irradiation, an additional mediumor high-dose UVB irradiation was re-irradiated for $30 \mathrm{~min}$. Subsequently, the cells were collected with $0.25 \%$ Trypsin and analyzed using a fluorescence spectrophotometer (F-2500; Hitachi, Tokyo, Japan) to detect the fluorescence of DCF inside the cells (excitation wavelength, $488 \mathrm{~nm}$; emission wavelength, $521 \mathrm{~nm})$.

RT-PCR analysis. Total RNA was extracted from exposed dorsal skin tissue and relative mRNA was normalized to $18 \mathrm{~s}$ rRNA. The primers used are listed in Table I.

Western blot analysis. A vertical slab gel with $12 \%$ polyacrylamide was used for protein electrophoresis. The transfer of proteins from the SDS-PAGE gels to the membrane was performed electrophoretically with some modifications using a Semi Dry Electroblotter (Sartorius) for $90 \mathrm{~min}$ with an electric current of $14 \mathrm{~V}$. Each membrane was treated with Block $\operatorname{Ace}^{\mathrm{TM}}(2.5 \%)$ for $60 \mathrm{~min}$ at $22^{\circ} \mathrm{C}$. The first immunological reaction was performed using a specific primary mouse monoclonal IgG 4HNE antibody (Abnova, Taipei, Taiwan, China), rabbit IgG MMP2 antibody (Proteintech Group Inc., Wuhan, China), mouse monoclonal IgG TIMP2 and TIMP4 antibodies (Abcam Trading Co. Ltd., Shanghai, China) in PBS containing $0.03 \%$ Tween-20 overnight at $4^{\circ} \mathrm{C}$. The second reaction was performed using HRP-conjugated anti-rabbit $\operatorname{IgG}$ for $1 \mathrm{~h}$ at $22^{\circ} \mathrm{C}$. The ECL Plus Western Blotting Detection System ${ }^{\mathrm{TM}}$ was used for enhanced chemiluminescence (ECL) detection of the protein bands.

SOD activity assay. SOD activity assay was performed as previously described (25). In brief, skin tissues were homogenized in cold buffer $(0.1 \mathrm{M}$ Tris/ $\mathrm{HCl}, \mathrm{pH} 7.4$ containing 
A

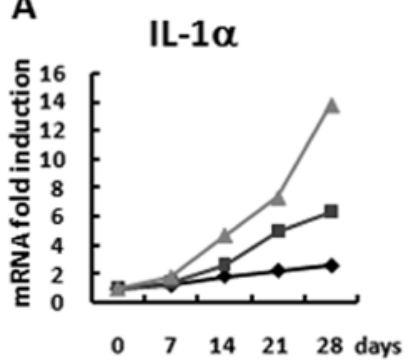

IL-1及

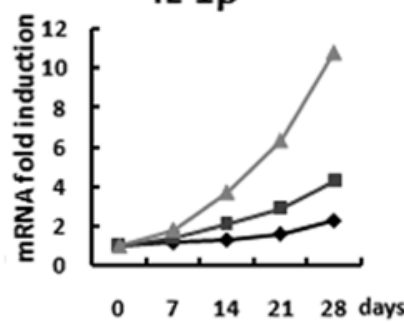

C
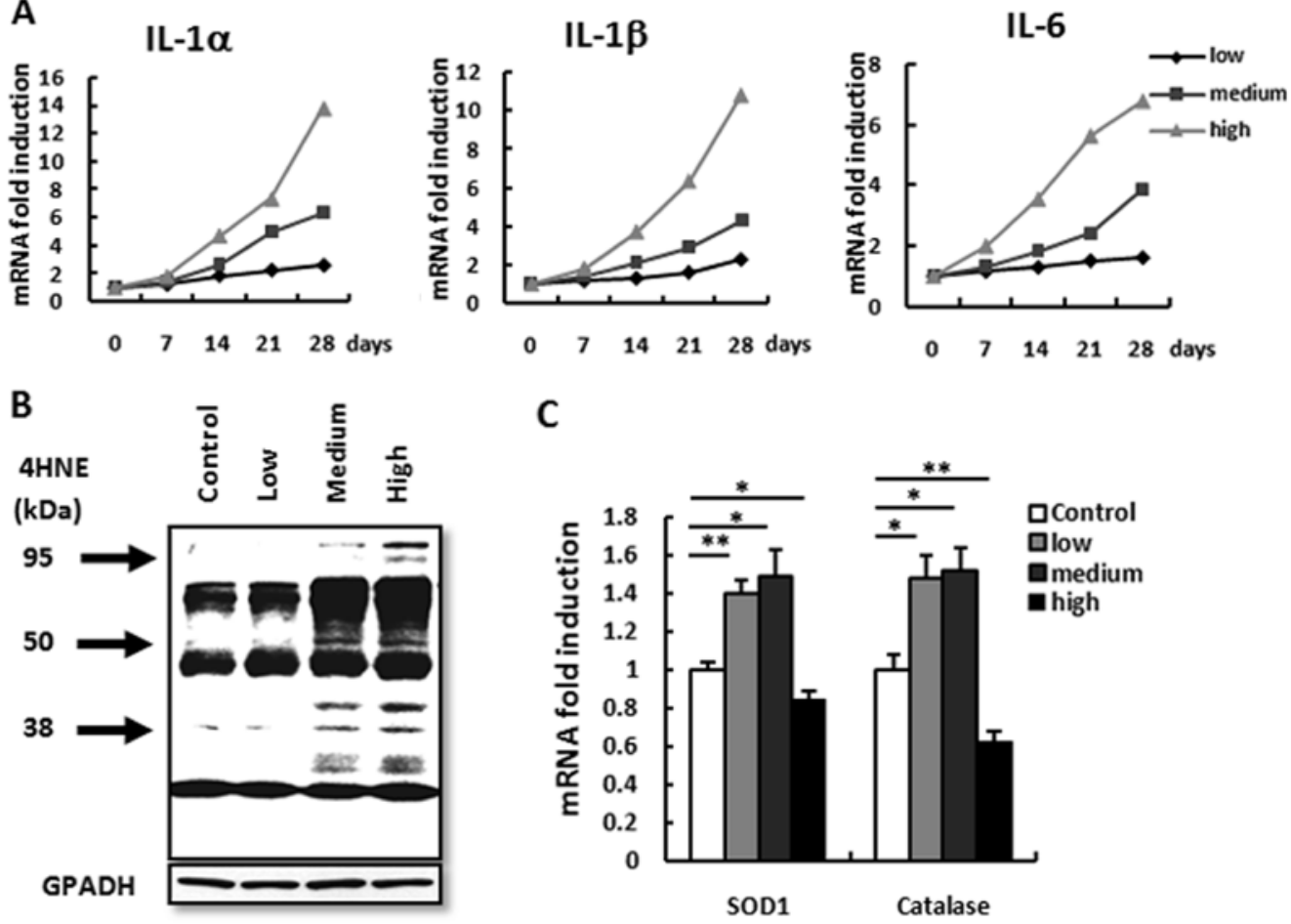

D

Control



Medium

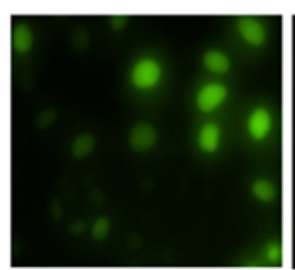

Low

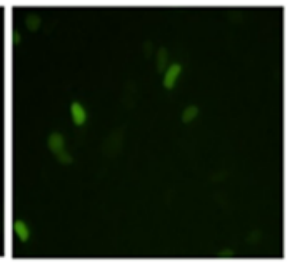

High

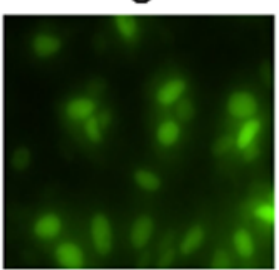



Figure 1. Different doses of UV irradiation induce different inflammation and ROS reactions. (A) Inflammatory cytokines IL-1 $\alpha$, IL-1 $\beta$ and IL-6 in mouse dorsal skin after low-, medium- and high-dose UV irradiation as determined by RT-PCR. Low-dose UV irradiation barely induced any inflammation during the 4-week time-course. However, both medium- and high-dose UV irradiation induced a dose-dependent gradually increase in inflammation. (B) ROS accumulation from mouse dorsal skin after 4 weeks of three doses of UV irradiation was detected by the 4HNE antibody. Low-dose UV irradiation induced no ROS accumulation, while medium- and high-dose UV irradiation induced a significant increase in ROS accumulation compared with the control group. (C) mRNA expression of SOD1 and catalase, two anti-ROS enzymes, from mouse dorsal skin were detected by RT-PCR after 4 weeks of UV irradiation. Both low- and medium-doses of UV irradiation induced elevated SOD1 and catalase mRNA expression, while high-dose UV irradiation induced a decrease in expression. (D) Intracellular (HacaT cells) ROS accumulation was estimated using the fluorescent dye $\mathrm{H}_{2}$-DCFDA after different doses of UV irradiation. Intracellular ROS accumulation was increased in a UV dose-dependent manner. Data are expressed as the mean \pm standard deviation $(\mathrm{n}=3)$. ${ }^{*} \mathrm{P}<0.05$ and ${ }^{* *} \mathrm{P}<0.01$ were considered to indicate a significant difference. ROS, reactive oxygen species.

$0.5 \%$ Triton X-100, $5 \mathrm{mM} \beta-\mathrm{ME}$ and $0.1 \mathrm{mg} / \mathrm{ml} \mathrm{PMSF})$ and homogenates were centrifuged at $14,000 \mathrm{x}$ g for $15 \mathrm{~min}$ at $4^{\circ} \mathrm{C}$. The resulting supernatant that contained cytosolic SOD was subjected to an SOD activity assay with a commercial SOD activity assay kit (Biovision, Palo Alto, CA, USA).

Evaluation of keratinocyte hyperplasia. Dorsal skin tissues were excised $24 \mathrm{~h}$ after the last irradiation, and each harvested tissue sample was fixed in formalin and embedded in paraffin.
Each sample was then cut into a section at $0.05-\mathrm{mm}$ intervals. Each section was then stained with H\&E staining. The thickness of the skin tissue keratinocytes was measured by SigmaPlot (Systat Software Inc. Chicago, IL, USA), and the average was calculated.

Gelatin zymography. To measure MMP2 activity, an extract of the supernatant of the skin tissue was collected. The gelatin zymography kit (Yagai Corporation, Tokyo, Japan) was used 

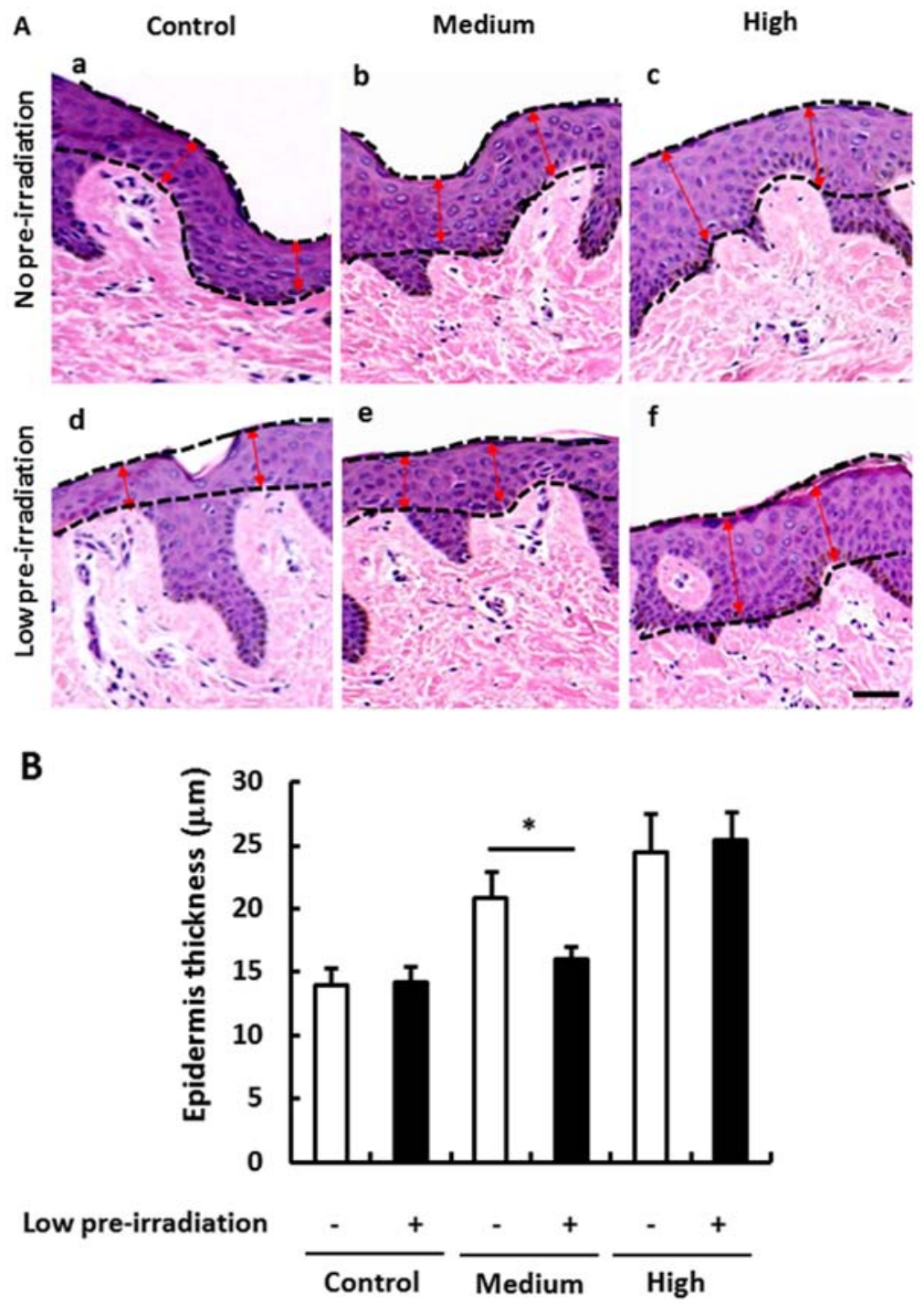

Figure 2. Low-dose pre-UV irradiation protects skin epithelium against medium-dose UV irradiation-induced hyperplasia, but not high-dose UV irradiationinduced hyperplasia. (A) H\&E-stained section of mouse dorsal skin after UV irradiation. Skin epithelium is marked by black lines and the red double headed-arrows indicate the thickness of the epithelium. The medium-dose UV irradiation group showed weak epithelial hyperplasia in the group treated with low pre-irradiation than in the group with no pre-irradiation ( $b$ and e). In contrast, in the high-dose UV irradiation groups, there were no changes after low pre-irradiation compared to that in the no pre-irradiation group (c and f). (B) Quantification of epithelium thickness in A. Data are expressed as the mean \pm standard deviation $(\mathrm{n}=3)$. $\mathrm{P}<0.05$ was considered to indicate a significant difference.

according to the manufacturer's instructions to perform gelatin zymography. In brief, $15 \mu \mathrm{l}$ samples of conditioned supernatant were subjected to electrophoresis through gels provided in the gelatin zymography kit. Each gel was washed twice, once with each type of washing buffer for $30 \mathrm{~min}$ and then incubated for $40 \mathrm{~h}$ at $37^{\circ} \mathrm{C}$ in the reaction buffer. Each gel was then stained with Coomassie blue and then destained three times so that the protein bands with gelatinolytic activity were easily identified.

Statistical analysis. Data are expressed as means \pm SEM of triplicate runs. Each experiment was repeated as least twice. The Student's t-test and analysis of variants were used to assess differences and $\mathrm{P}<0.05$ was considered to be significant.

\section{Results}

Effects of the different doses of UV irradiation on skin inflammation and ROS production. In order to confirm the role of various doses of UV irradiation on inflammation and ROS, we detected the time course of various classic inflammatory factors IL-1 $\alpha$, IL-1 $\beta$ and IL- 6 mRNA from mouse dorsal skin tissues exposed to the three doses of UV irradiation. It was observed that low-dose irradiation did not induce any inflammation after a 4-week exposure compared to that before irradiation. A medium- or a high-dose irradiation induced a very significant gradual increase compared to that before irradiation (Fig. 1A). ROS accumulation detected by $4 \mathrm{HNE}$ also showed a similar result. Low-dose irradiation barely induced any ROS accumulation, while medium- or high-dose irradiation induced a significant increase in ROS accumulation (Fig. 1B). This result is consistent with the results of the ROS accumulation detection in the HacaT keratinocyte cell line (Fig. 1D). However, different from inflammation, the difference in ROS accumulation induced between medium- and high-dose irradiation was not significant (Fig. 1B). This result forced us to consider that the imbalance of ROS accumulation and anti-ROS may be 
A







C

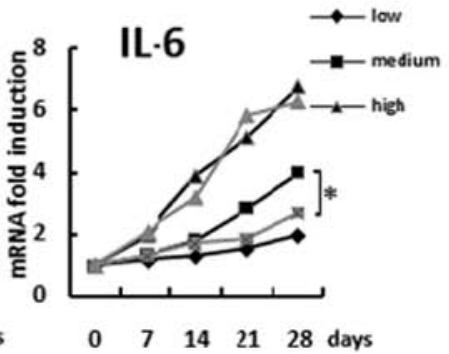

$\begin{array}{lllll}0 & 7 & 14 & 21 & 28 \\ \text { days }\end{array}$
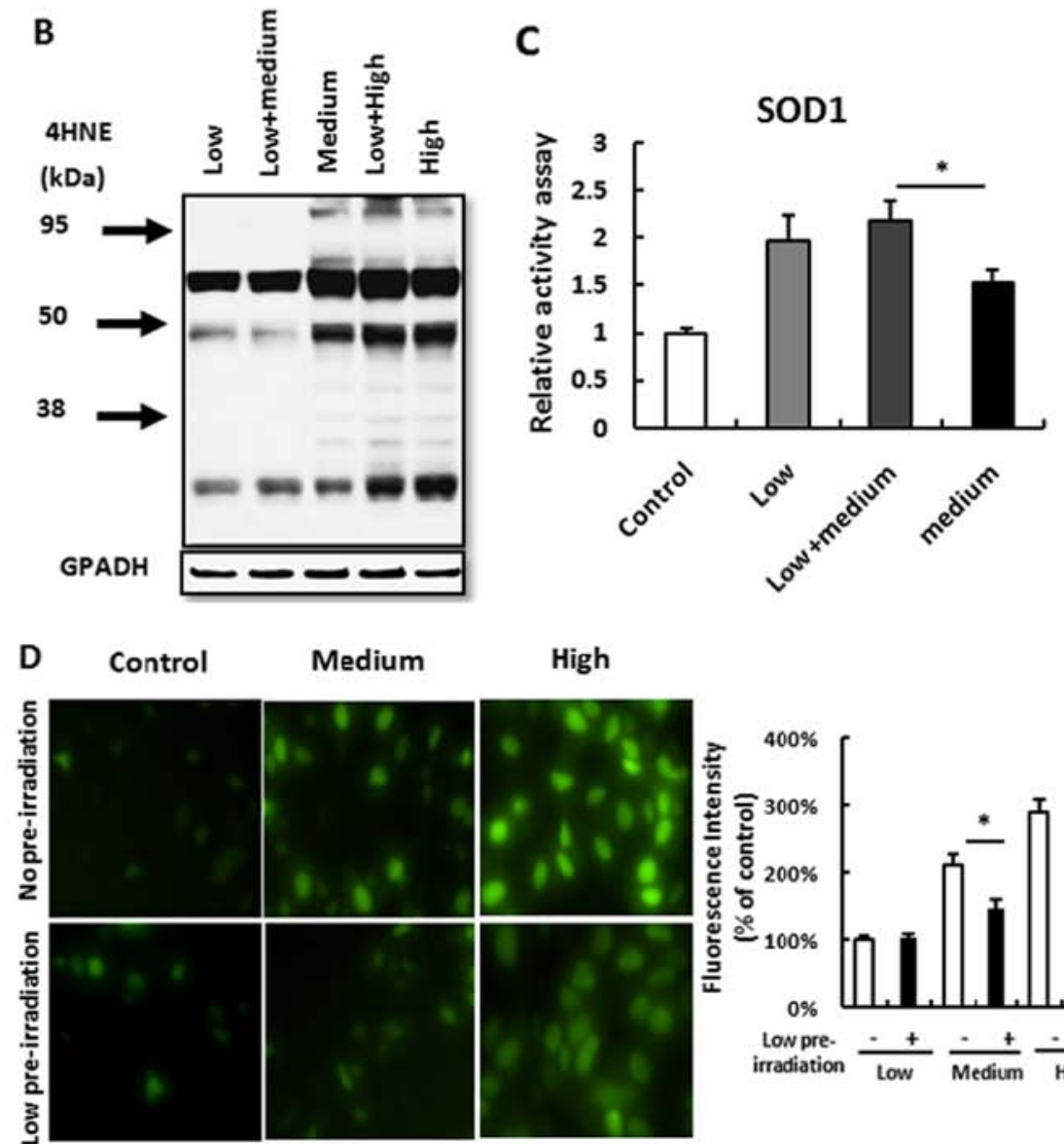

Medium

High


Figure 3. Low-dose pre-irradiation suppresses medium-dose UV irradiation-induced inflammation and ROS accumulation but not after high-dose UV irradiation. (A) mRNA expression levels of IL-1 $\alpha$, IL-1 $\beta$ and IL-6 from mouse dorsal skin were measured by RT-PCR. Low-dose pre-irradiated skin showed low inflammatory cytokine expression when compared with levels in the no pre-irradiated skin after medium-dose UV irradiation, but not after high-dose UV irradiation. (B) ROS accumulation from mouse dorsal skin detected by 4HNE antibody. Low-dose pre-irradiated skin showed a low ROS accumulation when compared with that with no pre-irradiated skin after medium-dose UV irradiation, but not after high-dose UV irradiation. (C) Activity of SOD1 was detected by the SOD activity assay kit. Low- but not medium-dose UV irradiation elevated SOD1 activity. (D) Intracellular (HacaT cells) ROS accumulation was estimated using the fluorescent dye $\mathrm{H}_{2}$-DCFDA. Low-dose pre-irradiated cells showed low ROS accumulation when compared with that in the no pre-irradiated cells after medium-dose UV irradiation, but not after high-dose UV irradiation. Data are expressed as the mean \pm standard deviation $(\mathrm{n}=3$ ). " $\mathrm{P}<0.05$ was considered to indicate a significant difference. ROS, reactive oxygen species.

the reason for the difference (26). Thus, we next detected the anti-ROS enzyme SOD1 and catalase. Both were increased in the low- and medium-dose irradiation groups but decreased in the high-dose irradiation group (Fig. 1C). These results indicate that although skin inflammation and ROS accumulation were correlated with the dose of UV irradiation, low-dose UV induced no inflammation and no ROS but increased anti-ROS ability, a potential protect effect.

Low-dose pre-irradiation protects skin epithelium against medium-dose UV irradiation-induced hyperplasia. Since low-dose irradiation presented a potential protective role, we aimed to ascertain whether low-dose UV irradiation may also suppress the medium- and high-dose induced skin epithelial proliferation and hypertrophy. Firstly, we confirmed that the skin epithelial hypertrophy was dependent on the dose of UV irradiation (Fig. 2Aa-c and B), while low-dose UV irradiation did not induce any hypertrophy compared with the no preirradiation group (Fig. 2Aa and d and B). However, compared to the medium-dose UV irradiation-induced epithelial hypertrophy (Fig. 2Aa and b and B), we found that medium-dose UV irradiation did not induce hypertrophy even in the low-dose 
A


C



B



Figure 4. Low-dose pre-irradiation increases TIMP2 consequently inhibiting MMP2 expression. (A) Skin MMP2 protein was detected by western blot analysis and quantified. (B) Skin MMP2 protein activity was detected by gelatin zymography and quantified. Low-dose pre-irradiation inhibited medium-dose UV irradiation-induced MMP2 protein expression and activity but did not inhibit high-dose UV irradiation-induced MMP2 expression and activity. (C) Skin TIMP2/4 protein was detected by western blot analysis and quantified. Low-dose pre-irradiation increased TIMP2 protein but decreased TIMP4 protein. Data are expressed as the mean \pm standard deviation $(n=3)$. ${ }^{*} \mathrm{P}<0.05$ and ${ }^{* *} \mathrm{P}<0.01$ was considered to indicate a significant difference.

pre-irradiation group (Fig. 2Ad and e and B). The inhibitory role was not observed when a high-dose UV irradiation was performed (Fig. 2Ac and $\mathrm{f}$ and $\mathrm{B}$ ). These results indicated an inhibitory role of low-dose UV irradiation on medium-dose UV irradiation-induced epithelial hypertrophy, but not on high-dose UV irradiation-induced hypertrophy.

Low-dose pre-irradiation suppresses medium-dose UV irradiation-induced inflammation and ROS accumulation. As skin inflammation and ROS may lead to epithelial proliferation and even skin tumors (27) and low-dose pre-irradiation protected skin epithelial proliferation, we aimed to ascertain whether low-dose pre-irradiation suppresses UV irradiation-induced inflammation and ROS. All the mice were administere a medium- or high-dose re-irradiation after a low-dose UV pre-irradiation. We found that low-dose pre-irradiation suppressed medium-dose irradiation-induced inflammation but not high-dose irradiation-induced inflammation (Fig. 3A). Paralleled with this result, low-dose irradiation suppressed medium-dose irradiation-induced ROS accumulation but not high-dose irradiation-induced ROS accumulation (Fig. 3B). The same result was also found in 
A


Figure 5. Conclusive conceptual schemes of the different doses of UV irradiation inducing different reactions in the ROS and TIMP2/MMP2 system. (A) Moderate UV-irradiation elevated TIMP2 and anti-ROS ability, which suppressed MMP2 and ROS accumulation. (B) When an individual is exposed to a relative excessive UV irradiation, although TIMP2 and anti-ROS ability are increased there is failure to suppress the further increase in MMP2 and ROS accumulation. (C) When an individual is exposed to a moderate level of UV irradiation, the TIMP2 and anti-ROS ability are elevated. Then the elevated TIMP2 and anti-ROS ability suppresses more ROS accumulation and MMP2 induced by higher UV irradiation. (D) Absolute excessive UV irradiation not only induces MMP2 and ROS accumulation, but also impairs TIMP2 and anti-ROS ability. ROS, reactive oxygen species.

the HacaT keratinocyte cell line culture. The medium-dose UV irradiated cells after low-dose pre-irradiation presented significant low fluorescence intensity compared with that in the group with no pre-irradiation, but this suppression was not found in the high-dose irradiation group (Fig. 3D).

Next, we aimed to ascertain why low-dose UV irradiation suppresses medium-dose irradiation-induced ROS accumulation. Although medium-dose irradiation induced anti-ROS SOD1 expression (Fig. 1C), medium-dose irradiation did not suppress ROS production (Fig. 1B). These results suggest that SOD1 activity was not enough to suppress extra ROS production. Thus, we measured SOD1 activity in skin tissue after irradiation. Confirming our hypothesis, SOD1 activity was decreased after medium-dose irradiation when compared with low-dose irradiation, but low-dose pre-irradiation induced elevated SOD1 activity and prevented SOD1 activity loss induced by medium-dose irradiation (Fig. 3C). These results indicated that low-dose irradiation may elevate SOD1 activation to suppress medium-dose irradiation-induced ROS production and inflammation.

Low-dose pre-irradiation increases TIMP2 to inhibit MMP2 expression. Although low-dose irradiation suppressed medium-dose UV irradiation-induced inflammation and ROS accumulation, which are the causes of skin epithelial hyper- plasia, we did not know whether low-dose irradiation also affects MMP2 and TIMPs which are the direct mechanisms of skin epithelial hyperplasia (28). We found that low-dose irradiation did not affects the MMP2 expression and its activation, however, low-dose irradiation inhibited the increase in MMP2 induced by medium- but not high-dose irradiation (Fig. 4A and B).

Next, we aimed to ascertain how MMP2 inhibitor, TIMP2 and TIMP4, are affected after low-dose irradiation. We found that TIMP2 was increased following single low-dose irradiation and then decreased following medium- and high-dose irradiation and TIMP4 was not significant altered following low-dose irradiation but increased following medium- and high-dose irradiation. However, after low-dose pre-irradiation, TIMP2 was highly expressed in the medium-dose irradiation group and TIMP4 was decreased. Both TIMP2 and TIMP4 were not significantly altered following high-dose irradiation (Fig. 4C). These results indicated that low-dose irradiation may elevate TIMP2 to inhibit MMP2 expression and activation but not TIMP4.

\section{Discussion}

In the present study, we demonstrated that a relative non-inflammation and non-ROS-inducing low-dose UV irradiation is a protective factor to suppress slight inflammation and ROS accumulation, which is induced by a medium-dose UV irradiation, further suppressing slight skin epithelial hyperplasia. This may be contributed to elevated SOD1 activity and suppressed skin epithelial MMP2 activity inhibited by increased TIMP2 activity. However, this type of suppression was not observed in high-dose UV irradiation-induced inflammation, ROS accumulation and skin epithelial hyperplasia with MMP2 activity. These results indicate that the protective role of low-dose UV irradiation is only limited to the most initial stage and for very slight lesions.

A relative low-dose UV irradiation induced no inflammation or ROS production (Fig. 1A and B), that was contributed to elevated anti-ROS enzymes (Fig. 1C). Although medium-dose UV irradiation also induced the same level of anti-ROS enzymes, which may not suppress the continuous accumulation of ROS. But with the increased intensity of UV irradiation, the anti-ROS system was absolutely damaged after high-dose UV irradiation (Fig. 1B and C). This indicates the dual character of UV irradiation on the ROS-antioxidant system $(29,30)$. It is very easy for us to understand how ROS are portrayed as detrimental, as evidenced by the notable trend in the use of dietary and cosmetic antioxidants (31). However, recently, it was reported that increased ROS promote longevity and metabolic health, which may be explained by mitochondrial hormesis (mitohormesis) $(32,33)$. ROS, derived from the mitochondrial electron transport system, may be necessary triggering elements for a sequence of events that result in benefits ranging from the transiently cytoprotective to organismal-level longevity. Basis on this concept, it is not difficult to understand that ROS also play an important role in the beneficial alterations in cellular physiology produced by caloric restriction, intermittent fasting, exercise and dietary phytonutrients (34). Thus, the balance of ROS and antioxidants seems to be a key element (30). 
As the dual character of ROS, in the present study was stimulated by UV irradiation and in light of the importance of the ROS-antioxidant balance, it is difficult to judge whether it is beneficial or not for health. However, it seemed that low accumulation of ROS induced by low-dose UV irradiation, although the low accumulation of ROS was not suppressed by elevated antioxidants (Fig. 1C and D), inhibited further inflammation and further ROS accumulation induced by medium-dose UV irradiation (Fig. 3A, B and D). In the present study, there was an interesting phenomenon displayed. Low-dose UV irradiation inhibited medium-dose UV irradiation. It may be explained that an accumulation of good ROS inhibited the accumulation of bad ROS or the increasing antioxidants (SOD and catalase) inhibited further ROS accumulation (35). We also found another interesting phenomenon that both low- and medium-dose UV irradiation induced the same level of SOD expression (Fig. 1C). However, low-dose UV irradiation induced SOD-suppressed ROS accumulation and a further ROS induced by medium-dose UV irradiation, while the same level of SOD induced by medium-dose UV irradiation did not suppress ROS accumulation (Figs. 1B and 3B). This was because SOD activity was increased after low-dose UV irradiation but damaged after medium-dose UV irradiation (Fig. 3C). The protective role of low-dose UV irradiation contributed to the increased antioxidant activity more than simple antioxidant expression.

As inflammation and ROS induce skin epithelial hyperplasia $(27,36)$, we also demonstrated that low-dose UV irradiation suppressed medium-dose UV irradiation induced skin epithelial hyperplasia (Fig. 2Ab and e), which was paralleled with its inhibition of inflammation and ROS accumulation. Furthermore, low-dose UV irradiation did not inhibit high-dose UV irradiation-induced inflammation and ROS, yet low-dose UV irradiation also did not inhibit high-dose UV irradiation-induced skin epithelial hyperplasia (Fig. 2Ac and f).

Skin epithelial hyperplasia, resulting from degradation of the basement membrane (BM) and extracellular matrix (ECM), is caused by epithelial cell proliferation and migration (37) and migration was reported to be correlated with the balance of MMP2 and its inhibitor TIMP2/4 $(38,39)$. Although MMP9, MMP7 and MMP13 were also reported to be correlated with keratoacanthomas and skin SCC $(40,41)$, we found that MMP2 was more strongly correlated with UV-induced skin epithelial hyperplasia (Fig. 4A and B). TIMP2 and TIMP4, well known as inhibitors of MMP2, presented different reactions to the increased MMP2 activity (Fig. 4C). Accompanied by decreased MMP2, TIMP2 was increased but TIMP4 was also decreased. This indicated that the TIMP2-MMP2 system more directly influenced UV irradiation-induced skin epithelial hyperplasia. It may also be because TIMP2-MMP2 and TIMP4-MMP2 are different balance systems as TIMP2 does not act synergistically with TIMP4 (42). However, according to the inhibitory efficiency to aggrecanase- 1 , it is possible that TIMP2 has a 5-fold stronger inhibitory activity than TIMP4 (43), since acidic residues of Glu and Asp in the TIMP2 C-terminal tail are important for binding to pro-MMP2, but these acidic residues are lacking in TIMP4, in which the stability of complex formation with the MMP2 hemopexin C domain is reduced (44). Thus, although TIMP2 was increased following medium-dose UV irradiation with pre low-dose UV irradiation, at this time TIMP4 presented a weak inhibitory activity due to its low expression.

In conclusion as shown in Fig. 5, we demonstrated that low-dose UV irradiation, which does not induce any inflammation, protected skin against medium-dose UV irradiation-induced inflammation and ROS and further inhibited skin epithelial hyperplasia by regulating the balance of the TIMP2/MMP2 pathway. The present study indicates that not all UV exposure is bad or carcinogenic and moderate UV irradiation also has a beneficial role for increasing resistance to prevent cancer.

\section{References}

1. Jerant AF, Johnson JT, Sheridan CD and Caffrey TJ: Early detection and treatment of skin cancer. Am Fam Physician 62: 357-368, 375-356, 381-352, 2000.

2. Marks R: An overview of skin cancers: Incidence and causation. Cancer 75: 607-612, 1995.

3. de Gruijl FD: Skin cancer and solar UV radiation. Eur J Cancer 35: 2003-2009, 1999.

4. Yoshikawa T, Rae V, Bruins-Slot W, Van den Berg JW, Taylor JR and Streilein JW: Susceptibility to effects of UVB radiation on induction of contact hypersensitivity as a risk factor for skin cancer in humans. J Invest Dermatol 95: 530-536, 1990.

5. de Gruijl FR, van Kranen HJ and Mullenders LH: UV-induced DNA damage, repair, mutations and oncogenic pathways in skin cancer. J Photochem Photobiol B 63: 19-27, 2001.

6. Melnikova VO and Ananthaswamy HN: Cellular and molecular events leading to the development of skin cancer. Mutat Res 571: 91-106, 2005

7. Clydesdale GJ, Dandie GW and Muller HK: Ultraviolet light induced injury: Immunological and inflammatory effects. Immunol Cell Biol 79: 547-568, 2001.

8. Terui T, Okuyama R and Tagami H: Molecular events occurring behind ultraviolet-induced skin inflammation. Curr Opin Allergy Clin Immunol 1: 461-467, 2001.

9. Mantovani A, Alleven P, Sica A and Balkwill F: Cancer-related inflammation. Nature 454: 436-444, 2008.

10. Diakos CI, Charles KA, McMillan DC and Clarke SJ: Cancer-related inflammation and treatment effectiveness. Lancet Oncol 15: e493-e503, 2014.

11. Bickers DR and Athar M: Oxidative stress in the pathogenesis of skin disease. J Invest Dermatol 126: 2565-2575, 2006.

12. Caricchio R, McPhie L and Cohen PL: Ultraviolet B radiationinduced cell death: Critical role of ultraviolet dose in inflammation and lupus autoantigen redistribution. J Immunol 171: 5778-5786, 2003.

13. Lawrence T, Willoughby DA and Gilroy DW: Anti-inflammatory lipid mediators and insights into the resolution of inflammation. Nat Rev Immunol 2: 787-795, 2002.

14. Holick MF: Vitamin D: importance in the prevention of cancers, type 1 diabetes, heart disease, and osteoporosis. Am J Clin Nutr 79: 362-371, 2004.

15. Holick MF: The vitamin D epidemic and its health consequences. J Nutr 135: 2739S-2748S, 2005.

16. Bikle DD: The vitamin D receptor: A tumor suppressor in skin. Adv Exp Med Biol 810: 282-302, 2014.

17. Gloster HM J and Neal K: Skin cancer in skin of color. J Am Acad Dermatol 55: 741-760, 2006.

18. Halder RM and Bang KM: Skin cancer in Blacks in the United States. Dermatol Clin 6: 397-405, 1988.

19. Koh D, Wang H, Lee J, Chia KS, Lee HP and Goh CL: Basal cell carcinoma, squamous cell carcinoma and melanoma of the skin: Analysis of the Singapore Cancer Registry Data 1968-1997. Br J Dermatol 148: 1161-1166, 2003.

20. Vermeer M, Schmieder GJ, Yoshikawa T, van den Berg JW, Metzman MS, Taylor JR and Streilein JW: Effects of ultraviolet B light on cutaneous immune responses of humans with deeply pigmented skin. J Invest Dermatol 97: 729-734, 1991.

21. Gallagher RP, Hill GB, Bajdik CD, Fincham S, Coldman AJ, McLean DI and Threlfall WJ: Sunlight exposure, pigmentary factors, and risk of nonmelanocytic skin cancer. I. Basal cell carcinoma. Arch Dermatol 131: 157-163, 1995. 
22. Rees JL: Genetics of hair and skin color: Annu Rev Genet 37: 67-90, 2003.

23. Jablonski NG: The evolution of human skin and skin color. Annu Rev Anthropol 33: 585-623, 2004.

24. Rastogi RP, Singh SP, Häder DP, and Sinha RP: Detection of reactive oxygen species (ROS) by the oxidant-sensing probe 2',7'-dichlorodihydrofluorescein diacetate in the cyanobacterium Anabaena variabilis PCC 7937. Biochem Biophys Res Commun 397: 603-607, 2010.

25. Luo JD, Wang YY, Fu WL, Wu J and Chen AF: Gene therapy of endothelial nitric oxide synthase and manganese superoxide dismutase restores delayed wound healing in type 1 diabetic mice. Circulation 110: 2484-2493, 2004.

26. Braga PC, Marabini L, Wang YY, Lattuada N, Calò R, Bertelli A, Falchi M, Dal Sasso M and Bianchi T: Characterisation of the antioxidant effects of Aesculus hippocastanum L. bark extract on the basis of radical scavenging activity, the chemiluminescence of human neutrophil bursts and lipoperoxidation assay. Eur Rev Med Pharmacol Sci 16 (Suppl 3): 1-9, 2012.

27. Mueller MM: Inflammation in epithelial skin tumours: Old stories and new ideas. Eur J Cancer 42: 735-744, 2006.

28. Graesslin O, Cortez A, Fauvet R, Lorenzato M, Birembaut $\mathrm{P}$ and Daraï E: Metalloproteinase-2, -7 and -9 and tissue inhibitor of metalloproteinase-1 and -2 expression in normal, hyperplastic and neoplastic endometrium: A clinical-pathological correlation study. Ann Oncol 17: 637-645, 2006.

29. Acker T, Fandrey J and Acker H: The good, the bad and the ugly in oxygen-sensing: ROS, cytochromes and prolyl-hydroxylases. Cardiovasc Res 71: 195-207, 2006.

30. Kawagishi $\mathrm{H}$ and Finkel T: Unraveling the truth about antioxidants: ROS and disease: finding the right balance. Nat Med 20 711-713, 2014.

31. Perera RM and Bardeesy N: Cancer: When antioxidants are bad. Nature 475: 43-44, 2011.

32. Ristow $\mathrm{M}$ and Zarse K: How increased oxidative stress promotes longevity and metabolic health: The concept of mitochondrial hormesis (mitohormesis). Exp Gerontol 45: 410-418, 2010.

33. Ristow M: Unraveling the truth about antioxidants: Mitohormesis explains ROS-induced health benefits. Nat Med 20: 709-711, 2014.

34. Tapia PC: Sublethal mitochondrial stress with an attendant stoichiometric augmentation of reactive oxygen species may precipitate many of the beneficial alterations in cellular physiology produced by caloric restriction, intermittent fasting, exercise and dietary phytonutrients: 'Mitohormesis' for health and vitality. Med Hypotheses 66: 832-843, 2006.
35. Klabunde RE and Anderson DE: Role of nitric oxide and reactive oxygen species in platelet-activating factor-induced microvascular leakage. J Vasc Res 39: 238-245, 2002.

36. Wullaert A, Bonnet $\mathrm{MC}$ and Pasparakis $\mathrm{M}: \mathrm{NF}-\kappa \mathrm{B}$ in the regulation of epithelial homeostasis and inflammation. Cell Res 21 : 146-158, 2011.

37. Thiery JP: Epithelial-mesenchymal transitions in tumour progression. Nat Rev Cancer 2: 442-454, 2002.

38. Benson JM, Seagrave J, Weber WM, Santistevan CD, Grotendorst GR, Schultz GS and March TH: Time course of lesion development in the hairless guinea-pig model of sulfur mustard-induced dermal injury. Wound Repair Regen 19: 348-357, 2011.

39. Teulière J,Faraldo MM, Deugnier MA, Shtutman M, Ben-Ze'ev A, Thiery JP and Glukhova MA: Targeted activation of beta-catenin signaling in basal mammary epithelial cells affects mammary development and leads to hyperplasia. Development 132: 267-277, 2005.

40. Coussens LM, Tinkle CL, Hanahan D and Werb Z: MMP-9 supplied by bone marrow-derived cells contributes to skin carcinogenesis. Cell 103: 481-490, 2000.

41. Kuivanen TT, Jeskanen L, Kyllönen L, Impola U and Saarialho-Kere UK: Transformation-specific matrix metalloproteinases, MMP-7 and MMP-13, are present in epithelial cells of keratoacanthomas. Mod Pathol 19: 1203-1212, 2006.

42. Toth M, Bernardo MM, Gervasi DC, Soloway PD, Wang Z, Bigg HF, Overall CM, DeClerck YA, Tschesche $\mathrm{H}$ and Cher ML: Tissue inhibitor of metalloproteinase (TIMP)-2 acts synergistically with synthetic matrix metalloproteinase (MMP) inhibitors but not with TIMP-4 to enhance the (Membrane type 1)-MMP-dependent activation of pro-MMP-2. J Biol Chem 275: 41415-41423, 2000.

43. Hashimoto G, Aoki T, Nakamura H, Tanzawa K and Okada Y: Inhibition of ADAMTS4 (aggrecanase-1) by tissue inhibitors of metalloproteinases (TIMP-1, 2, 3 and 4). FEBS Lett 494: 192-195, 2001.

44. Kai HS, Butler GS, Morrison CJ, King AE, Pelman GR and Overall CM: Utilization of a novel recombinant myoglobin fusion protein expression system to characterize the tissue inhibitor of metalloproteinase (TIMP)-4 and TIMP-2 C-terminal domain and tails by mutagenesis. The importance of acidic residues in binding the MMP-2 hemopexin C-domain. J Biol Chem 277: 48696-48707, 2002. 